\title{
Accuracy of selected indicators in leaking for the management of dengue haemorrhagic fever
}

\author{
Ranasinghe K N P ${ }^{1}$, Dilhari K A A ${ }^{2}$, Dissanayake ${ }^{1}$, Vigneswaran $\mathrm{K}^{1}$, Chinthaka A M $\mathrm{H}^{1}$, Mujahieth, $\mathrm{M}^{1}{ }^{1}$
}

Journal of the Ceylon College of Physicians, 2018, 49, 25-29

\begin{abstract}
Aims: Conventional clinical and laboratory parameters for the management of dengue haemorrhagic fever (DHF) were validated using ultrasound scan (USS) as the gold standard.
\end{abstract}

\begin{abstract}
Methods: Clinically suspected 184 patients with platelet count $<100,000$ were enrolled in the study.

Results: USS evidence of leaking was observed in $48 \%$ of the sample. When Hct rises $>10 \%$ and $>20 \%$ were used as a predictor of leaking, sensitivity and specificity of 30\%, 82\% (PPV $60 \%$, NPV 56\%) and 11\%, 94\% (PPV 63\% and NPV 54\%) were observed. Liver tenderness and gall bladder wall oedema (wall thickness $>6 \mathrm{~mm}$ ) had sensitivity of $28 \%, 91 \%$ and specificity of $83 \%$ and $94 \%$ as predictors of leaking. Haematocrit rise $>10 \%$ during any stage of critical phase associated with 94\% sensitivity and 37\% specificity (PPV 46\%, NPV 92\%) in prediction of complications.
\end{abstract}

Conclusions: Rising HCT, liver tenderness and clinical detection were found to be weak predictors of onset of leaking. HCT rise was a strong predictor of complications during the leaking phase.

\section{Introduction}

Dengue fever (DF) is caused by any of four closely related viruses, or serotypes: dengue virus $1-4^{1,2}$. Infection with one viral serotype does not induce protective immunity against other dengue viral serotypes $^{3}$. Secondary dengue infections increase the

\footnotetext{
${ }^{1}$ Base Hospital, Horana, Sri Lanka.

${ }^{2}$ Department of Microbiology, Faculty of Medical Sciences, University of Sri Jayewardenepura, Sri Lanka.
}

Corresponding author: Ranasinghe $K N P$

E-mail: nilranasinghe@gmail.com risk of dengue haemorrhagic fever (DHF). The proportion of dengue patients going in to DHF, and the outcome greatly varies according to virus serotype, geographic region and host factors ${ }^{4}$.

Third space fluid accumulation (TSFA) or leaking is the hallmark of $\mathrm{DHF}^{4}$, where in ward close monitoring is essential in order to prevent death. Accurate detection of the onset of leaking phase is one of the most difficult tasks in day to day practice in dengue fever management in busy medical units. Detection of leaking in a dengue patient is mainly a clinical diagnosis. This clinical diagnosis is supported by several investigations and clinical signs such as platelet count (less than $100 \times 10^{3} / \mu \mathrm{l}$ ), Hematocrit $(\mathrm{Hct})$ rise and liver tenderness ${ }^{4,5}$. Detection of ascites and pleural effusion by ultrasound scan (USS) is considered as the gold standard in identification of leaking as all other predictors of leaking are inaccurate, variable and subjective ${ }^{5}$.

The hematocrit changes can be due to many other conditions like dehydration, smoking, hypoxia etc ${ }^{5}$. Over hydration with oral or parental fluids on the other hand artificially reduce the rising $\mathrm{Hct}^{5}$. Concealed bleeding among dengue shock patients (in leaking phase) is associated with inappropriately low Hct and results in improper management. Based on clinicians observations, a rising WBC also is suggested as an indicator of leaking but this is not properly validated. Although liver tenderness is a simple clinical sign during examination, it is very nonspecific and present in many other causes of hepatitis.

Facilities for ultra sound scan for every dengue patient is not available in many medical units in Sri Lanka. Therefore, clinicians have to depend on their clinical experience and other subjective investigations such as rising Hct to identify and manage DHF in day to day practice. Furthermore, many of ultrasonically detected dengue leaking patients eventually recover without causing any of significant complications and only few patients experience serious outcomes. Therefore, it is important to find out clinical and laboratory parameters to monitor patients during leaking phase for early identification of complications and to reduce the mortality rate and the burden on health care professionals. 


\section{Methods}

The study was a prospective cross-sectional study carried out for a period of a year (2015-2016) in medical wards in Base Hospital, Horana. Ethical approval was granted by the National Institute of Health Science (NIH), Sri Lanka (NIHS/ERC/16/29). One hundred and eighty four dengue suspected patients were included for the study diagnosed according to 2012 National guideline ${ }^{3}$. Informed written consent was obtained from the patients.

Case definition - Dengue illness (DF/DHF) was considered in the differential diagnosis of patients presenting with acute onset of fever with two or more following: headache, retro-orbital pain, myalgia, arthralgia, rash (diffuse, erythematous, macular) hemorrhagic manifestation (petechiae, positive tourniquet test) with leukopaenea $\left(<5 \times 10^{3} / \mu \mathrm{l}\right)$, platelet count, $<150 \times 10^{3} / \mu \mathrm{l}$ and rising of haematocrit by $5-10 \% 5$. Such patients were clinically diagnosed as suspected dengue patients and selected for this study after obtaining the written consent when their platelet count was $<100 \times 10^{3} / \mu \mathrm{l}$.

Study population was subjected to ultrasound scan twice daily. Abdominal ultrasound was performed on a Toshiba Xario 100 (TUS-X100) unit, equipped with a 6C1 wide bandwidth (3.0-5.0 MHz) convex probe and $11 \mathrm{~L} 4$ wide bandwidth ( 7 to $12, \mathrm{MHz}$ ) linear probe. The abdominal ultrasound was done in all cases after 4 or more hours of fasting for better visualization of the gall bladder. A consultant radiologist who is a member of this study performed all the serial ultrasound examinations till the end of critical phase. Hct and full blood count were measured after 3-4 hours of ultra sound scan. Clinical diagnosis of leaking was obtained after discussion with dengue management clinical team irrespective of the result of ultra sound scan.

Demographic and clinical details of the patients were recorded using a pre tested questionnaire. The statistical analysis was carried out by using the software, Statistical Package for Social Sciences (SPSS Inc., Chicago, IL) version 17.0. Descriptive statistics were represented as a percentage (\%) value. The statistical tests for qualitative variables were carried out using the chi-square test $\left(\chi^{2}\right.$ test). All these tests were two sided. The level of significance was taken at $5 \%(p<0.05)$.

\section{Results}

Of the 184 patients 113 (61\%) were females and 71 (39\%) were males. The mean age of the study cohort was $40 \pm 17.3$ years. Ultra sound scan evidence of leaking was observed in $48 \%$ (88/184) of dengue suspected patients who had a platelet count less than $100 \times 10^{3} / \mu \mathrm{l}$.

The duration of fever when patients start leaking were tabulated in table 1.

More than $10 \%$ Hct rise from base line was observed in $30 \%(26 / 88)$ of patients and over $20 \%$ rise was seen in $11 \%(10 / 88)$ of ultrasonically confirmed leaking patents in the first four hours of leaking (Table 2).

When $>10 \%$ Hct rise from baseline was used as a predictor of leaking, against USS scan within four hours it showed $30 \%$ sensitivity and $82 \%$ specificity (PPV 60\%, NPV 56\%) whilst > 20\% Hct rise showed $11 \%$ sensitivity and 94\% specificity (PPV 63\% and NPV 54\%).

Liver tenderness was observed in $28 \%$ (25/88) of patients within early hours of leaking. The overall clinical judgment of leaking irrespective of the ultrasound scan was observed in $29.5 \%$ (26/88) of leaking patients (Table 3).

Liver tenderness had a sensitivity and specificity of $28 \%$ and $83 \%$. Clinical judgment of leaking had a sensitivity and specificity of $30 \%$ and $89 \%$.

Gallbladder wall oedema was considered when gall bladder wall thickness was over $6 \mathrm{~mm}$ for this study. Gallbladder wall oedema was observed in a total of 86 patients. Of them 80 patients had leaking. When gallbladder wall thickness of more than $6 \mathrm{~mm}$ was considered as a predictor of leaking a sensitivity and specificity of 91\% and 94\% (PPV 93\% and NPV 92\%) were observed (Table 4).

Although nearly $50 \%$ patients had leaking according to ultrasound scan $1 / 3$ of them were managed with hourly fluid replacement not exceeding $100 \mathrm{ml}$ at any stage without a rise in Hct, reduction of urine output or any other complications. Serial ultrasound scans did not show any evidence of progression of leaking. There were 9 patients with pre shock and all were having leaking. They were successfully managed with fluid boluses (both normal saline and dextran). Hematocrit rise $>10 \%$ during any stage of critical phase was associated with $94 \%$ sensitivity and $37 \%$ specificity (PPV 46\%, NPV 92\%) in the prediction of complications (shock, pre shock, reduction of urine output for less than $0.5 \mathrm{ml}$ per kilogram body weight for two hours, and those who needed fluid boluses > 200 per hour). The association between complications and hematocrit rise $>10 \%$ is represented in Table 5 . 
Table 1. The duration of fever days when patients start leaking (total leaking patients $n=88$ )

\begin{tabular}{lcccccccc}
\hline Number of fever days & D-1 & D-2 & D-3 & D-4 & D-5 & D-6 & D-7 & D-8 \\
Number of patients & 0 & 02 & 30 & 36 & 12 & 06 & 02 & 0 \\
\hline
\end{tabular}

D: Day

Table 2. Association between HCT rise and the leaking

\begin{tabular}{|c|c|c|c|c|c|}
\hline & & \multicolumn{3}{|c|}{ Leaking } & \multirow{2}{*}{$P$ value } \\
\hline & & Yes & No & Total & \\
\hline \multirow{4}{*}{ Hct rise } & Nil & 62 & 79 & 141 & \multirow{4}{*}{0.162} \\
\hline & $10-20 \%$ & 16 & 11 & 27 & \\
\hline & $>20 \%$ & 10 & 6 & 16 & \\
\hline & Total & 88 & 96 & 184 & \\
\hline
\end{tabular}

Table 3. Association between liver tenderness, clinical judgment and the leaking

\begin{tabular}{|c|c|c|c|c|c|}
\hline & & \multicolumn{3}{|c|}{ Leaking } & \multirow{2}{*}{$P$ value } \\
\hline & & Yes & No & Total & \\
\hline \multirow{3}{*}{ Liver tenderness } & No & 63 & 80 & 143 & \multirow{3}{*}{0.056} \\
\hline & Yes & 25 & 16 & 41 & \\
\hline & Total & 88 & 96 & 184 & \\
\hline \multirow{3}{*}{$\begin{array}{l}\text { Clinical judgment } \\
\text { of leaking }\end{array}$} & No & 62 & 85 & 147 & \multirow{3}{*}{0.002} \\
\hline & Yes & 26 & 11 & 37 & \\
\hline & Total & 88 & 96 & 184 & \\
\hline
\end{tabular}


Table 4. Association between gall bladder wall oedema and the leaking

\begin{tabular}{l|c|crr|c}
\hline \multicolumn{2}{c|}{} & \multicolumn{3}{c|}{ Leaking } & \multirow{2}{*}{ P value } \\
\cline { 3 - 5 } \multicolumn{2}{c|}{} & Yes & No & Total & \\
\hline \multirow{3}{*}{ Gall bladder wall } & No & 8 & 90 & 98 & \\
oedema $>\mathbf{6} \mathbf{~ m m}$ & Yes & 80 & 6 & 86 & \multirow{2}{*}{0.000} \\
& Total & 88 & 96 & 184 & \\
\hline
\end{tabular}

Table 5. Association between complications and hematocrit rise $>10 \%$

\begin{tabular}{l|c|ccc|c}
\hline \multicolumn{2}{c|}{} & \multicolumn{3}{|c|}{ Hematocrit rise $>10 \%$} & \multirow{2}{*}{ P value } \\
\cline { 3 - 5 } \multicolumn{2}{c|}{} & $>10 \%$ & $<10 \%$ & Total & \\
\hline \multirow{3}{*}{ Complication } & No & 2 & 23 & 25 & \\
& Yes & 33 & 39 & 72 & 0.000 \\
& Total & 35 & 62 & 97 & \\
\hline
\end{tabular}

Out of 88 patients with leaking 50 (57\%) were afebrile when leaking started, and 19 (21.5\%) had fever for less than a day during the critical phase. Another $19(21.5 \%)$ leaking patients had continuous fever during the leaking phase.

In the study population any detectable change in pattern of white blood cells (WBCs) was not observed at the beginning of critical phase.

\section{Discussion}

Nearly $50 \%$ of study population had USS evidence of leaking. This is a relatively higher proportion compared to previous reports 6 . A previous study done in (2004-2006) Sri Lanka had reported nearly 16\% DHF cases among 357 DF patients ${ }^{6}$. Another study done in Sri Lanka in 2013, including 102 dengue patients with platelet count less than $100 \times 10^{3} / \mu$ l found $41 \%$ had leaking ${ }^{7}$. This is evidence for variable and unpredictable nature of dengue infections and increasing trend of secondary infections over time ${ }^{7}$.

In this study, we selected clinically suspected dengue patients with platelet count less than $100 \times 10^{3} / \mu \mathrm{l}$.
All of them underwent serial USS scans twice daily followed by haematocrit assessment and clinical assessment to identify leaking. Conventional methods such as rising Hct, liver tenderness and clinical judgement were weak predictors of onset of leaking. Although they showed low sensitivity (30\%) it had relatively higher specificity. It is clear that conventional methods are weak predictors of leaking and can miss many DHF patients in early hours of onset of the leaking.

In the present study gall bladder wall oedema was considered when thickness of the gall bladder exceeds $>6 \mathrm{~mm}$. Gall bladder wall thickness of more than 3 $\mathrm{mm}$ is a nonspecific finding in many conditions including dengue fever as well as $\mathrm{DHF}^{8}$. It has been previously reported that around 30\% DF patients can present with gall bladder wall oedema $>3 \mathrm{~mm}$ and wall thickness increase markedly among patients with severe dengue $e^{8,9,10}$. However when gall bladder wall oedema is more than $6 \mathrm{~mm}$ it associated with 91\%, $94 \%$ sensitivity and specificity respectively. Based on the results gall bladder wall oedema exceeding $>6$ $\mathrm{mm}$ was found to be a good predictor of leaking in DHF rather than $3 \mathrm{~mm}$. 
In the study over one third of patients with ultra sound evidence of leaking didn't show any progression in leaking or complications such as reduction in urine output or pre shock. All of them were managed with hourly fluid requirement not exceeding $100 \mathrm{ml}$ and all of them were clinically well during entire critical phase. Therefore it is not clear whether these patients belong to a separate category "pseudo leakers" or real DHF patients with mild short lasting fluid leakage. In a busy medical ward these patients have to be monitored hourly as such patients are categorized under DHF. Based on the results of the present study although serial ultrasound scans are found to be very useful in detecting the onset of leaking it seems there were possible overestimations.

Hct rise $>10 \%$ during critical phase was found to be a strong predictor of complications as it showed 94\% sensitivity in predicting the patients with complications including those who needed fluid boluses more than $200 \mathrm{ml}$ an hour. Therefore, rising haematocrit can be used to monitor patients during critical phase.

\section{Conclusion}

Based on the finding of this study conventional methods such as rising haematocrit were found to be weak predictors of detection of leaking. However they can be successfully used to monitor DHF patients during critical phase to identify complications.

\section{References}

1. Malavige GN, Fernando S, Fernando DJ, Seneviratne SL,
Dengue viral infections. Postgraduate Medical Journal 2004; 80(948): 588-601.

2. Sirisena PD, Noordeen F. Evolution of dengue in Sri Lankachanges in the virus, vector, and climate. International Journal of Infected Diseases 2014; 19: 6-12.

3. Kularatne SAM. Dengue fever. British Medical Journal 2015; 351: 4661.

4. Mallhi $\mathrm{TH}$, Khan $\mathrm{AH}$, Adnan AS, Sarriff $A$, Khan $\mathrm{YH}$, Jummaat F. Clinico-laboratory spectrum of dengue viral infection and risk factors associated with dengue hemorrhagic fever: a retrospective study. BMC Infectious disease 2015; 15: 399.

5. Gunawardane N, Wijewickrama A, Dissanayake U, Sellahewa K, Tissera H. Guidelines on management of dengue fever and dengue hemorrhagic fever in adults. Colombo: Ministry of Health, Sri Lanka. 2012.

6. Kanakaratne N, Thevanesam V, Messer WB, Ranawaka G, Shahani A, De SilvaAM, Gunasekera M. Clinical features of hospitalized dengue patients in Sri Lanka from 2004 to 2006. Sri Lanka Journal of Infectious Diseases 2012; 1(2): 9-18.

7. Premarathne R, Ragupathy A, Mithinda JKND, De Silva HJ. Timing predictors, and progress of third space fluid accumulation during preliminary phase fluid resuscitation in adult patients with dengue. International Journal of Infectious Diseases 2013; 17e505-e509.

8. Adam A, Dixon AK, Gillard JH, Schaefer-Prokop C, Grainger RG. Grainger \& Allison's Diagnostic Radiology. 6th Edition. ISBN: 9780702042959. 2014.

9. Parmar JP, Mohan C, Vora M. Patterns of gall bladder wall thickening in dengue fever: A mirror of the severity of disease. Ultrasound International Open 2017; 3(2): E76.

10. Setiawan MW, Samsi TK, Pool TN, Sugianto D, Wulur H. Gallbladder wall thickening in dengue hemorrhagic fever: an ultrasonographic study. Journal of Clinical Ultrasound 1995; 23(6): 357-62. 